\title{
Marcadores de dano oxidativo na doença de Fabry
}

\section{Markers of oxidative damage in Fabry's Disease}

\author{
Marinês Ghizi Souza ${ }^{1}$ \\ Tamires Marques de $\mathrm{Abreu}^{2}$ \\ Jéssica Pereira Marinho ${ }^{1}$ \\ Luiz Fernando Lopes ${ }^{2}$ \\ Izabel Cristina Teixeira Proença ${ }^{1}$ \\ John Thomas Weriang² \\ Cláudia Funchal ${ }^{3}$ \\ Caroline Dani ${ }^{3}$ \\ Alexandre Silva de Mello $^{3}$
}

\section{RESUMO}

A doença de Fabry (DF) é uma doença de herança genética ligada ao cromossomo X, com mutações no gene que codifica a enzima lisossômica Alfa-galactosidase A ( $\alpha$-gal A). A baixa atividade enzimática da $\alpha$-gal A, resulta no acúmulo progressivo do substrato Globotriaosilceramida (Gb3) nas células, prejudicando a função do sistema metabólico e podem estar associado a alteração das espécies reativas de oxigênio e do sistema de defesa antioxidante que contribue para o estresse oxidativo. 0 objetivo do estudo foi testar, através da análise de amostras de plasma, avaliando a peroxidação lipídica analisada por substâncias reativas ao ácido tiobarbitúrico (TBARS), dano protéico por ensaio de carbonila, defesas antioxidantes não enzimáticas pelo teor de sulfidrila (SH), defesas enzimáticas antioxidantes por superóxido dismutase (SOD) e catalase (CAT). Analisamos dois grupos, sendo um com 14 pacientes diagnosticados com DF que não receberam tratamento prévio, comparados com 13 indivíduos do grupo controle.A atividade da enzima $\alpha$-gal A foi medida no plasma e leucócitos. Os resultados no grupo da SOD e CAT apresentou uma correlação positiva entre os dois grupo. Porem não mostraram diferenças significativas $(p<0,05)$ nos níveis de TBARS, carbonila, sulfidrila e SOD. Em conclusão, os parametros estudados manteram uma constante nos níveis de defesa enzimática e não enzimática, sem nenhum impacto sobre a peroxidação lipídica e danos às proteínas. Consideramos que outros fatores no plasma de paciente com Fabry podem contribuir para o extresse oxidativo.

\section{PALAVRAS-CHAVE}

Doença de Fabry; Alfa-galactoseA; Extresse Oxidativo

\footnotetext{
${ }^{1}$ Mestrandas do Programa de Pós-Graduação em Biociências e Reabilitação - Centro Universitário Metodista - IPA.

${ }^{2}$ Graduandos de Biomedicina do Centro Universitário Metodista - IPA.

${ }^{3}$ Docentes do PPG em Biociências e Reabilitação do Centro Universitário Metodista - IPA.
} 


\section{ABSTRACT}

The Fabry Disease is a inherit disease associated to the $\mathrm{X}$ chromosome, with a gene mutation which codifies the lysosomal enzyme Alfa-galactosidase A ( $\alpha$-gal A). The $\alpha$-gal A low enzymatic activity results in a progressive accumulation of substrate Globotriaosilceramide (Gb3) in the cells, damaging the metabolic system functions and can be related to the change of the oxygen reactive species and the antioxidant defense system, which contributes to the oxidative stress. The oxidative damage aims to prejudicial implications in the cellular components as lipids peroxidation, protein and damage. This study aimed to test through plasma sample analysis, evaluate the lipidic peroxidation analysed by responsive substances to the Thiobarbituric acid (TBARS), protein damage test by carbonyl, non-enzymatic antioxidant defense by the levels of sulphydryl (SH), enzymatic antioxidant defense by superoxide dismutase (SOD) and catalase (CAT). Two groups were analyzed, one group with 14 patientsdiagnotised with Fabry disease that did not received the previous treatment compared to another group with 13 patients of the control group.The activity of $\alpha$-gal A enzyme was measured in plasma and leukocytes. The results in the SOD and CAT group showed a positive correlation between the two groups. However, they did not show significant differences $(p<0.05)$ in the levels of TBARS, carbonyl, sulfhydryl and SOD. In conclusion, the studied parameters maintained a constant in the enzymatic and non-enzymatic defense levels, with no impact on lipid peroxidation and protein damage. Can be considered that other factors in the plasma of Fabry disease patient can contribute to the oxidative stress.

\section{KEYWORDS}

Fabry disease; Alfa-galactosidaseA e Oxidative stress 


\section{INTRODUÇÃo}

As Doenças Lisossômicas de Depósito (DLDs) representam um grupo com mais de 50 doenças metabólicas genéticas, que ocorrem pela deficiência da atividade de enzimas lisossômicas especificas ou defeito nas proteínas lisossômicas não enzimáticas, resultando no acúmulo do substrato não degradado nos lisossomos, causando uma disfunção celular, em tecidos e órgãos (LAMPE et al 2013). São consideradas as doenças raras com alterações hereditárias em genes que codificam enzimas lisossômicas, de origem autossômicas recessivas, com uma prevalência estimada de 1 em cada 5.000 nascidos vivos (PLATT, 2014). No Brasil, de acordo com a Organização Mundial De Saúde (OMS), consideram as doenças raras aproximadamente cerca de 13 milhões de indivíduos no país ( INTERFARMA, 2018).

A Doença de Fabry (DF) entra no grupo das DLDs causadas pela baixa ou ausência de atividade da enzima Alfa-galactosidase A ( $\alpha$-Gal A), que gera um acúmulo progressivo de glicoesfingolipídeos, em particular globotriaosilceramida (Gb3), nos lisossomos podem contribuir para danos tecidual causando disfunção em múltiplos órgãos. A deposição lissosomal de substrato Gb3, está concentrado principalmente nas células endoteliais, rins, coração e células nervosas, gerando uma série de sintomas como acroparestesia, angioqueratomas, córnea verticillata e fibrose tecidual irreversível nos rins e coração (DESNICK, 2001; BOGGIO,2009). 0 diagnostico da doença deve ser reconhecido com brevidade, certamente a demora do diagnostico, contribui para progressão da doença elevando a morbimortalidade ( BERNARDES et al, 2020). A ativação do processo inflamatorio na DF, pode estrar associado a outras evoluções patológicas como o aumento das móleculas pró oxidantes (ROZENFELD \& FERIOZZI, 2017).

Um estudo demonstrou que o armazenamento intracelular de Gb3 em pacientes com DF acarretou um aumento significativo na geração de espécie reativa de oxigênio (ERO), que pode induzir ao estresse oxidativo (EO) nas células endoteliais vasculares (SHEN, 2008). Além do acúmulo de Gb3, outros mecanismos importantes são conhecidos, como elevado dano oxidativo às proteínas e lipídios, acompanhados por menores defesas antioxidantes e maiores citocinas pró-inflamatórias, tendo uma importância na fisiopatologia da DF e que podem contribuir para a progressão da doença (BIANCINI, 2012; BIANCINI, 2016).

Os mecanismos de defesa antioxidante são responsáveis por manter as concentrações de oxidante em nível baixo, assim como reparando o dano celular causado pelo ERO, espécie reativa de nitrogênio (ERN) em busca de equilíbrio homeostático (SALVADOR, 2004). Os antioxidantes podem ser enzimáticos ou não enzimáticos e a sua principal função é impedir ou reduzir os danos causados às células pelas ERO, na tentativa de evitar ao máximo que ocorra o estresse oxidativo (HALLIWELL, 1999). Dentre os antioxidantes enzimático destacam-se o Superóxido Dismutase (SOD), Catalase (CAT), Sistema GlutationaPeroxidase (GPX) e GlutationaRedutase (GR) os antioxidantes não enzimáticos podem ainda ser classificado em antioxidantes endógenos e exógenos (VASCONCELOS et AL, 2007).

Neste estudo, investigamos a relação das ERO na DF, analisando os marcadores de EO em amostras de pacientes (n=14) com DF comparando com o grupo controle ( $\mathrm{n}=13)$. 0 objetivo de testar a utilização de substâncias reativas ao ácido tiobarbitúrico (TBARS), conteúdo total de Sulfidrila (SH), Carbonila, Superóxido Dismutase (SOD) e Catalase (CAT), medidos no plasma na DF e do grupo controle (GC))

\section{MÉTODOS}

\section{Pacientes e controles}

Esta pesquisa se caracteriza por um estudo experimental, retrospectivo, advindos de um banco de amostras biológicas. Onde cabe destacar que não foi necessária uma nova coleta sanguínea para a realização destes ensaios, uma vez que o volume de amostra (sangue) prevista para o diagnóstico foi possível para realização e inclusão destes ensaios, utilizando material que seria congelado e, posteriormente, descartado.

Resumidamente, o material chegará no laboratório de Doenças Lisossômicas de Depósito, do Departamento de Bioquímica da UFRGS para diagnóstico, onde após a utilização das amostras (soro/plasma) para os ensaios bioquímicos e confirmação diagnóstica, foram armazenadas a $-20^{\circ} \mathrm{C}$, até o dia da análise dos parâmetros de estresse oxidativoconstantes nesta pesquisa.

Foram obtidas amostras de $9 \mathrm{~mL}$ de sangue que foram coletadas diretamente de 14 pacientes previamente 
diagnosticados com DF e de 13 indivíduos não portadores da doença caracterizando o grupo controle (GC). 0 sangue total heparinizado foi centrifugado e submetido a uma técnica de separação (SKOOG, 1956) para obtenção de plasma e leucócitos. A medida da $\alpha$-Gal A em plasma foi realizada de acordo com a técnica de (DESNICK et al, 1973). Já para leucócitos, a medida foi realizada de acordo com (DAITX et al, 2012). O diagnóstico dos pacientes com DF foi realizado na Universidade Federal do Rio Grande do Sul (Porto Alegre, RS, Brasil).

0 cálculo do tamanho da amostra para comparação de médias com diferentes variâncias foi feito, estabelecendo o nível de significância a $5 \%$ e a potência em 90\%. Para isso, usamos o software estatístico MiniTab® 15. 0 cálculo indicou 13 sujeitos por grupo para um total de 26 amostras. Esta pesquisa faz parte do projeto aprovado pelo Comitê de Ética e Pesquisa - IPA, sob número de parecer: 1.290 .503 .

\section{ATIVIDADES ENZIMÁTICAS}

\section{Atividade de $\alpha$-galactosidase A ( $\alpha$-Gal A)}

A atividade da enzima $\alpha$-gal A foi determinada através de ensaios fluorométricos de acordo com (DESNICK et al, 1973) para plasma e leucócitos. Uma placa de 96 poços, de acordo com (DAITX et al, 2012). utilizaram o substrato artificial 4-metilumbeliferyl- $\alpha$-Dgalactoside, e também o inibidor da isoenzima alfa-galactosidase B,N-acetil-D-galactosamina, ambas fornecidas pela Sigma (St. Louis, MO, EUA, www.sigma. com). As amostras foram incubadas em uma agitação incubadora a $37^{\circ} \mathrm{C}$ e a fluorescência foi lida num leitor de microplacas fluorômetro a $365 \mathrm{~nm}$ de excitação e $450 \mathrm{~nm}$ de emissão. Uma solução com $10 \mathrm{mmol} / \mathrm{l}$ de substrato diluído em 0,5 mol / l acetato de sódio foi preparado para os ensaios de plasma. $200 \mathrm{mmol} / \mathrm{l}$ de $\mathrm{N}$-acetil-Dgalactosamina foi diluído nesta solução. Vinte e cinco microlitros da solução contendo substrato e inibidor e o mesmo volume de amostra foram adicionados a cada poço. Após incubação por $2 \mathrm{~h} 30^{\circ} \mathrm{C}, 200$ $\mu \mathrm{l}$ de tampão glicina a $1 \mathrm{~mol} / \mathrm{l}(\mathrm{pH} \mathrm{10,4)}$ foram adicionados a todos os poços e $25 \mathrm{~mL}$ de amostra de plasma foram adicionados aos espaços em branco. Todos os ensaios foram utilizado a curva de Michaelis-Menten (curva de substrato) usando concentrações de substrato variando de 1 a $15 \mathrm{mmol} / \mathrm{l}$ para plasma.

\section{PARÂMETROS DO ESTRESSE OXIDATIVO}

\section{Substâncias reativas ao ácido tiobarbitúrico (TBARS)}

Como índice de peroxidação lipídica, foi utilizado na produção de TBARS durante uma reação de aquecimento ácido, que é amplamente adotada como um método sensível para medir a peroxidação lipídica, como descrito anteriormente por (WILLS, 1996). As amostras foram agitadas por uma breve perda de tempo com $10 \%$ de ido tricloroacético (TCA) e 0,67\% de ido tiobarbitúrico (TBA) e depois aquecidas num banho de água a ferver durante 15 min em tubos fechados. Os TBARS foram determinados por absorbância a $535 \mathrm{~nm}$. Os resultados foram expressos como nmol / mg de proteína.

\section{Níveis de medição de proteínas modificadas oxidativamente}

0 dano oxidativo às proteínas foi avaliado pela determinação dos grupos carbonila com base em sua reação com dinitrofenilhidrazina (DNPH), como descrito anteriormente por (LEVINE et al, 1990). A DNPH reage com proteínas carbonilas para formar hidrazonas, que podem ser medidas espectrofotometricamente. Primeiramente, $500 \mu \mathrm{L}$ de $10 \mathrm{mM}$ de DNPH em $2 \mathrm{M}$ de $\mathrm{HCl}$ foram adicionados à temperatura ambiente, por $1 \mathrm{~h}$, com vortex a cada 10-15 min. Em seguida, $500 \mu \mathrm{L}$ de TCA a $20 \%$ foram adicionados e os tubos foram misturados e centrifugados por $3 \mathrm{~min}$. 0 sobrenadante foi então descartado, e os pellets foram lavados três vezes com $1 \mathrm{~mL}$ de etanol-acetato de etila (1:1) para remover o reagente livre. Após a centrifugação, a proteína precipitada foi redissolvida em 0,6 mL de solução de guanidina. As proteínas foram dissolvidas por $15 \mathrm{~min}$ a $37^{\circ} \mathrm{C}$. 0 material insolúvel foi removido por centrifugação numa microcentrífuga durante 3 min. A absorvância foi lida a $370 \mathrm{~nm}$. Quantidades iguais de amostras de proteína sem DNPH foram usadas como controles. Os resultados foram expressos em nmol / mg de proteína.

\section{Medição de grupos sulfidrilas totais (SH)}

O ensaio SH se na redução do ácido 5,5-ditio-bis (ácido 2-nitrobenzóico) (DTNB) por tióis, gerando um derivado amarelo (TNB) cuja absorção é medida espectrofotometricamente a $412 \mathrm{~nm}$. DTNB $0,1 \mathrm{mM}$ foi adicionado a $120 \mu \mathrm{L}$ das amostras. Isto foi seguido por uma 
incubação de 30 minutos à temperatura ambiente em um quarto escuro. A absorção foi medida a $412 \mathrm{~nm}$. 0 teor de sulfidrilas é inversamente correlacionado ao dano oxidativo às proteínas. Os resultados foram relatados em nmol / mg de proteína (AKSENOV et al, 2001).

\section{Medida da atividade da enzima antioxidante}

Atividade SOD foi determinada por um método espectrofotométrico, medindo a inibição da taxa de formação de adrenocromo a $480 \mathrm{~nm}$ (espectrofotómetro SP-2200, Bioespectro) em meio contendo 1 adrenalina $\mathrm{mM}$ e $50 \mathrm{mM}$ de glicina (BANNISTER, 1987). Os resultados foram expressos em USOD/mg de proteína. 0 método utilizado para determinar a actividade de CAT foi descrito por (AEBI, 1984) e determina a taxa de $\mathrm{H}_{2} \mathrm{O}_{2}$ a degradação, medindo a absorvância a 240 nm (espectrofotómetro SP-2200, Bioespectro). Os resultados foram expressos como UCAT / mg de proteína.

\section{Determinação de proteínas}

A concentração de proteína foi determinada de acordo com o método descrito por (LOWRY et al, 1951).

\section{Análise estatística}

Os dados foram avaliados por meio do teste $\mathrm{T}$ de Student, seguido do teste de Levene, utilizado para comparar os resultados da análise do plasma com os dos pacientes com DF e GC. A análise foi realizada por meio do pacote estatístico SPSS, versão 17.0 (SPSS Inc., Chicago, IL, EUA). 0 nível de significância foi estabelecido em $p<0,05$. Os dados são relatados como média $\pm \mathrm{SE}$. Os valores foram comparados utilizando testes não pa- ramétricos, como o teste U de MannWhitney. Correlações entre variáveis foram realizadas através de Teste de Spearman. 0 valor da significância estatística foi de

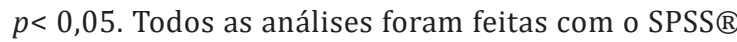
22.0 for Windows ${ }^{\circledR}$ (IBM Inc., Armonk, NY). Na análise das amostras dos pacientes em Plasma (nmol/h/mL) e Leucócitos (mMol/h/mg de proteína), foram considerados não paramétricos e apresentados através de mediana (valor mínimo - valor máximo). Porém os valores de referência são apresentados como Intervalo de Confiança (média \pm Desvio Padrão (Tabela 1).

\section{RESULTADOS}

Um total de 14 pacientes com DF e 13 do GC foram incluídos neste estudo. A idade média foi de 36,77 £16,82 para o DF e 24,11 $\pm 19,25$ para o GC. Após a coleta da amostra, mensuramos a análise enzimática, para confirmar que a amostra era de pacientes com DF . Na análise das amostras dos pacientes em Plasma (nmol/h/mL) e Leucócitos (mMol/h/mg de proteína), foram considerados não paramétricos e apresentados através de mediana (valor mínimo - valor máximo). Porém os valores de referência são apresentados como Intervalo de Confiança (média \pm Desvio Padrão (Tabela 1). Não foram observadas diferenças estatísticas entre os grupos DF e GC para os ensaios de TBARS, carbonil, sulfidrila e SOD..A análise paramétrica, foi realizada através do teste $t$ de amostras independentes, com resultado não significativa. Na CAT foi realizado um teste não paramétrico, demonstrado como mediana (intervalo interquartil), onde não se obteve diferença significativa como apresentado na (Tabela 2).

Tabela 1 - Análise enzimática dos pacientes com Doença de Fabry

\begin{tabular}{lccc}
\hline & DF $(\mathrm{n}=14)$ & Valores de referência & Ponto de Corte \\
\hline Plasma & $0,15(0,02-0,64)$ & ND $-4,43(6,02 \pm 2,84)$ & 4,16 \\
Leucócitos & $3,27(1,75-5,08)$ & $2,16-8,83(5,12 \pm 3,39)$ & 10,18 \\
\hline
\end{tabular}

Fonte: Elaborado pelos autores, 2018 
Tabela 2 - Análise paramétrica e não paramétrica realizada com teste t dos pacientes com doença de Fabry DF e Grupo Controle GC

\begin{tabular}{lccc}
\hline & DF $(\mathrm{n}=14)$ & GC $(\mathrm{n}=13)$ & P \\
\hline TBARS $^{*}$ & $2,27 \pm 1,57$ & $2,98 \pm 1,16$ & 0,126 \\
Carbonil $^{\#}$ & $16,38 \pm 9,63$ & $16,80 \pm 4,58$ & 0,868 \\
Sulfidril $^{\#}$ & $5,28 \pm 1,61$ & $6,32 \pm 2,97$ & 0,238 \\
SOD $^{\#}$ & $1,78 \pm 1,50$ & $3,21 \pm 3,12$ & 0,129 \\
CAT $^{*}$ & $4,19(2,14-5,46)$ & $6,22(3,90-13,62)$ & 0,228 \\
\hline
\end{tabular}

\#Análise paramétrica; *Análise não paramétrica

Fonte: Elaborado pelos autores, 2018

Entretanto, os resultados mostraram uma correlação plásmatica positiva entre SOD e CAT entre pacientes com Fabry e amostras controle: $r=0,51, p=0,008$ correlações entre variáveis foram realizadas, teste de Sperman, conforme demonstrado na Figura 1.

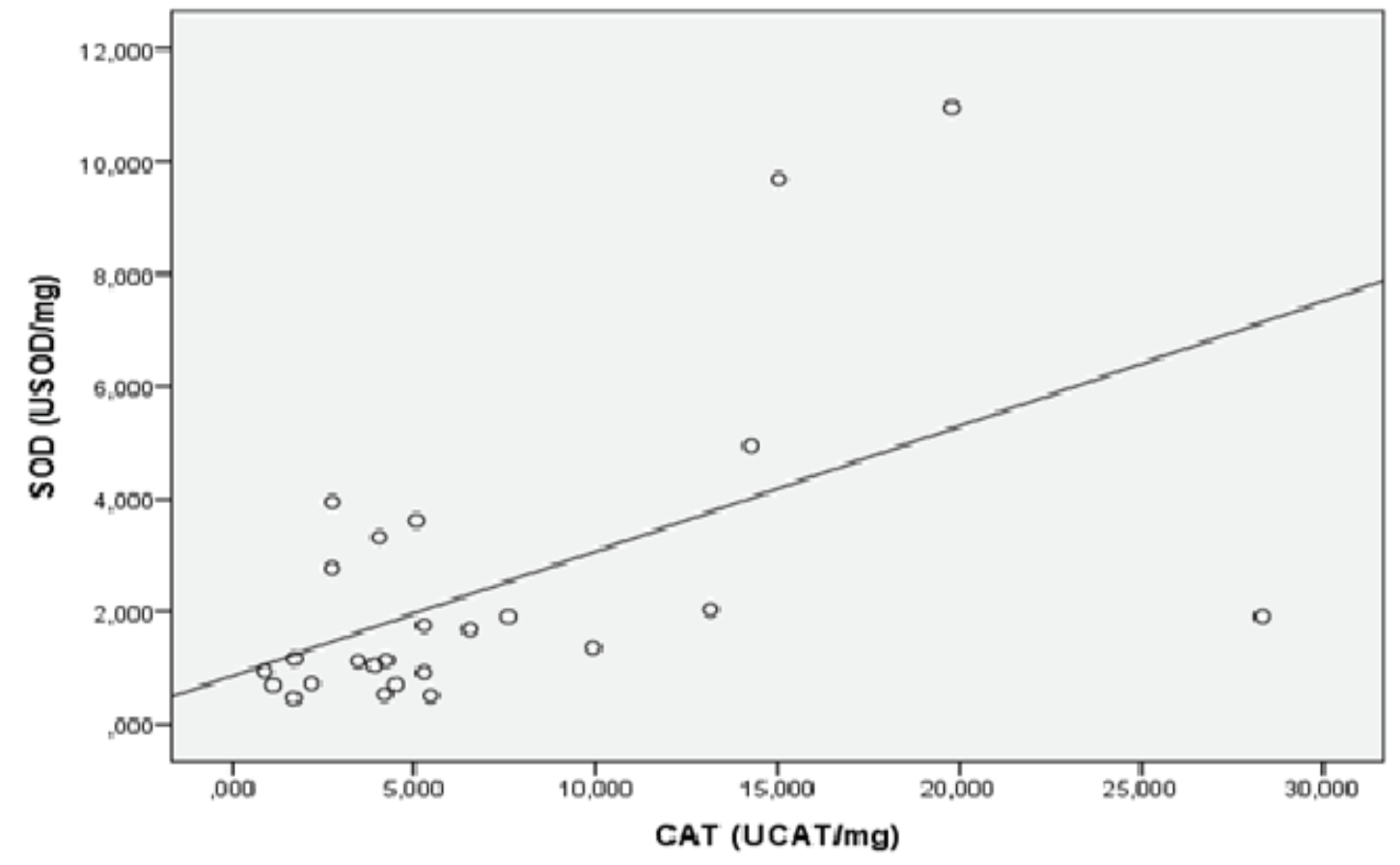

Figura:1Correlação entre SOD e CAT

Fonte: Dados de pesquisa (2018). 


\section{DISCUSSÃo}

As DLDs são alterações genéticas que implicam na deficiência de enzimas, gerando um acúmulo de substrato não degradado dentro dos lisossomos, o que causa disfunção celular de múltiplos órgãos (GIESELMANN, 1995). Alguns estudos mostram que os lisossomos são suscetíveis e tem condições favoráveis no processo do EO como o peróxido de hidrogênio que entra no compartimento lisossômico formando os radicais hidroxila ou radicais de ferro colocando em risco os lisossomos, causando a desestabilização e ruptura da membrana celular levando à apoptose ou necrose da célula (TERMAN, 2006; BRUNK,et al 2001).

Entre as DLD's encontramos a DF que se caracteriza pela atividade insuficiente da enzima lisossômica $\alpha$-gal A, resultando em uma concentração progressiva de glicoesfingolipídeos principalmente o acréscimo de substrato Gb3 nos lisossomos levando a uma disfunção ou danos celulares (DESNICK, 2001). Na DF o acúmulo do substrato $\mathrm{Gb} 3$ pode estar relacionado com outros processos fisiológicos colaborando para o avanço da doença, como a inflamação crônica (DE FRANCESCO, 2013; PEREIRA, 2013; ROZENFELD, FERIOZZI, 2017), a produção de espécies reativas (SHEN, 2008), o estresse oxidativo (BIANCINI, 2012).

0 aumento das espécies reativas (ER) e as diminuições do sistema de defesa antioxidante são fatores que contribuem para o estresse oxidativo, demonstrando envolvimento em várias fisiopatologias das DLD's como na Doença de Gaucher tipo I, (MELLO et al, 2015), e na Mucopolissacaridose (MPS) tipo IVA (DONIDA et al, 2015). No entanto, a produção descontrolada de ER resulta em dano oxidativo, alterando sua função e causando centenas de disfunções celulares (HALLIWELL et al 2007), como a peroxidação dos lipídios de membrana e agridem às proteínas dos tecidos, às enzimas, carboidratos e DNA (BARREIROS et al, 2006; CELI, 2010; OLIVEIRA \& SCHOFFEN, 2010). Para evitar os danos celulares deve haver um equilíbrio metabólico no nosso sistema dos pro-oxidante e dos antioxidantes (VASCONCELOS et al, 2007).

O tratamento da DF normalmente tem a indicação por meio da terapia de reposição enzimática e tem o objetivo de evitar ou remover depósitos de GB3 (BERNARDES et al, 2020)
Um estudo com pacientes com DF sob tratamento de reposição enzimática demonstrou diminuição das defesas antioxidantes e maior dano oxidativo a proteínas e lipídios, acompanhado por níveis aumentados de citocinas pró-inflamatórias, quando comparados aos controles (BIANCINI et al, 2012). A concentração acumulada de Gb3 induz o aumenta das ERO, em células endoteliais de Fabry cultivadas, indicando um mecanismo potencial de envolvimento vascular na doença de Fabry (SHEN, 2008).

A oxidação e fragmentos dos ácidos graxos podem produzir aldeído como malonaldeído MDA (um indicador de peroxidação lipídica), medido na reação TBARS (BONNES \& GUÉRIN, 1992). Neste estudo, os dados encontrados nos níveis de TBARS no plasma, não houve diferença estatisticamente significativa nos dois grupos estudados, corroborando com a literatura em doenças DLD`s, como na doença de Gaucher tipo I (MELLO et al, 2015). Um estudo apresentou nível maior de peroxidação lipídica em pacientes com Fabry antes e depois do TRE (BIANCINI, 2016).

O grupo carbonila está sendo aplicado como um indicador do dano oxidativo das proteínas (CHEVION et al, 2000). A relação do grupo carbonila e das proteína está relacionado a numerosos distúrbios patológicos, incluindo: artrite reumatóide, doença de Alzheimer, síndrome do desconforto respiratório, doença de Parkinson e aterosclerose (DE ZWART, 1999).Entretanto, não observamos alterações entre os valores dos grupos deste parâmetro estudado.

O grupo dos tióis são derivados orgânicos do enxofre, identificados pela presença de um grupo de sulfidríla presentes em sistemas biológicos, realizando várias funções, sendo fundamental na composição das defesas antioxidante, como, a proteção das células através da glutationa (GSH) que envolve o grupo sulfidrila (SH). 0 estado redox dos tióis nas células, protege a proteína celular, que contém oxidação excessiva de SH. Este sistema inclui baixo peso molecular nos grupos de doadores de SH e enzimas que podem catalisar a redução dos grupos SH de proteínas e pró-oxidantes (ZUGNO, 2008). Os resultados de sulfidrila, desta pesquisa, não demonstraram diferenças estatisticas entre DF e GC. Esses dados corroboram com a literatura que não houve diferença no grupo dos tiois (sulfidrila), realizado em pacientes com Fabry( BIANCINI, 2012; BIANCINI, 2016). 
Os antioxidantes enzimáticos celulares estudados, SOD e CAT apresentam a primeira linha endógena para neutralizar os danos oxidativos do metabolismo intracelular em seres humanos. A SOD tem a finalidade de reduzir os ânions superóxidos formando peróxido de hidrogênio $\left(\mathrm{H}_{2} \mathrm{O}_{2}\right)$ e na sequência a CAT responsável pela degradação do $\mathrm{H}_{2} \mathrm{O}_{2}$ formando água e oxigênio molecular (BARREIROS et al, 2006). As alterações do sistema antioxidante podem causar aumento de $\mathrm{H}_{2} \mathrm{O}_{2}$ ou de um produto de sua decomposição presente no citosol e nas mitocôndrias, o local da formação de grande parte das ER (SALVADOR 2004; VALKO, 2007). O peróxido de hidrogênio com outro componente químico causa danos extremante perigosos para a célula como na reação de $\mathrm{Fe}_{2}+\mathrm{H}_{2} \mathrm{O}_{2}$ que produz o radical hidroxila $\left(\mathrm{OH}^{-}\right)$chamada reação de Fenton (HALLIWELL, 2006). $\mathrm{O} \mathrm{H}_{2} \mathrm{O}_{2}$ pode induzir o dano ao DNA devido á formação de $\mathrm{OH}^{-}$, tanto em amostras controle como em DF (BIANCINI 2015). Em outro estudo pacientes DF em tratamento de reposição enzimática apresentaram uma redução nas defesas antioxidantes e aumento do dano oxidativo a proteinas e lipídos seguido de maior nivel de citocinas pró-inflama- torias quando comparados ao grupo controle. A diminuição de antioxidante glutationa GSH refletiu no aumento de SOD/CAT, indicando provavél aumento de peroxido de hidrogênio livre para oxidar moléculas biológicas na DF (BIANCINI, 2012).

Entretanto, nosso estudo mostrou que os marcadores antioxidantes SOD e CAT apresentaram um crescimento linear dentre os grupos estudados, apresentando uma correlação positiva no plasma, mas não encontramos uma diferença estatisticamente significativa quando analisamos estes parâmetros separadamente.

\section{CONCLUSÃO}

O estudo mostroua análise dos marcadores no plasma de pacientes com DF e do GC. Obtivemos uma correlação positiva entre Superóxido Dismutase (SOD) e Catalase (CAT) que foi observada entre os dois grupos desta pesquisa. No entanto, os parâmetros do conteúdo total de sulfidrila (SH), ácido tiobarbitúrico (TBARS) e carbonila não apresentaram diferenças significativas após as realizações dos ensaios e as análises estatística. 


\section{REFERÊNCIAS}

AEBI, Hugo. [13] Catalase in vitro. In: Methods in enzymology. Academic Press, 1984. p. 121-126.

AKSENOV, Michael Y.; MARKESBERY, William R. Changes in thiol content and expression of glutathione redox system genes in the hippocampus and cerebellum in Alzheimer's disease. Neuroscience Letters, v. 302, n. 2-3, p. 141-145, 2001.

BANNISTER, Joe V.; CALABRESE, Lilia. Assays for superoxide dismutase. Methods of Biochemical Analysis, p. 279-312, 1987.

BARREIROS, A.L.; DAVID, J.M.; DAVID, J.P. Estresse oxidativo: Relação entre geração de espécies reativas e defesa do organismo. Química Nova, v. 29, n. 1, p. 113-123, 2006.

BERNARDES, Thaíza Passaglia; FORESTO, Renato Demarchi KIRSZTAJN, Gianna Mastroianni. Fabry disease: genetics, pathology, and treatment. Revista da AssociaçãoMédicaBrasileira, v. 66, p. s10-s16, 2020.

BIANCINI, Giovana B. et al. Globotriaosylceramideis correlated with oxidative stress and inflammation in Fabry patients treated with enzyme replacement therapy. BiochimicaetBiophysicaActa (BBA)-Molecular Basis of Disease, v. 1822, n. 2, p. 226-232, 2012.

BIANCINI, GiovanaBrondani et al. Biomolecules damage and redox status abnormalities in Fabry patients before and during enzyme replacement therapy. ClinicaChimica Acta, v. 461, p. 41-46, 2016.

BIANCINI, Giovana Brondani et al. DNA damage in Fabry patients: An investigation of oxidative damage and repair. Mutation Research/Genetic Toxicology and Environmental Mutagenesis, v. 784, p. 31-36, 2015.

BOGGIO, Paula et al. Fabrydisease. Anais brasileiros de dermatologia, v. 84, n. 4, p. 367-376, 2009.

BONNES-TAOUREL, Dominique; GUÉRIN, Marie-Christine; TORREILLES, Jean. Is malonaldehyde a valuable indicator of lipid peroxidation?. Biochemical pharmacology, v. 44, n. 5, p. 985-988, 1992.

BRUNK, Ulf T.; NEUZIL, Jiri; EATON, John Wallace. Lysosomal involvement in apoptosis. Redox Report, v. 6, n. 2, p. 91-97, 2001.

CELI, P. O papel do estresse oxidativo na saúde e produção de pequenos ruminantes. RevistaBrasileira de Zootecnia, v. 39 (suplemento especial), p. 348-363, 2010.

CHEVION, M.; BERENSHTEIN, E.; STADTMAN, E. R. Human studies related to protein oxidation: protein carbonyl content as a marker of damage. Free radical research, v. 33, p. S99108,2000
DAITX, Vanessa Vitcoski et al. Comparison between alpha-galactosidaseA activity in blood samples coletei on filter paper, leukocytes and plasma. Clinical biochemistry, v. 45, n. 15, p. 1233-1238, 2012.

DE FRANCESCO, Pablo N. et al. Fabry disease peripheral blood immune cells release inflammatory cytokines: role of globotriaosylceramide. Molecular genetics and metabolism, $v$. 109, n. 1, p. 93-99, 2013.

DE ZWART, Loeckie L. et al. Biomarkers of free radical damage: applications in experimental animals and in humans. Free Radical Biology and Medicine, v. 26, n. 1-2, p. 202-226, 1999.

DESNICK, R. J.; JOANNOU, Y. A.; ENG, C. M. Galactosidase-A deficiency: Fabry disease: nature of the accumulated glycosphingolipids. The metabolic and molecular basis of inherited disease. New York, NY: McGraw-Hill, p. 3733, 3774, 2001.

DESNICK, Robert J. et al. Fabry's disease: enzymatic diagnosis of hemizygotes and heterozygotes: $\alpha$-galactosidase activities in plasma, serum, urine, and leukocytes. The Journal of laboratory and clinical medicine, v. 81, n. 2, p. 157-171, 1973.

DONIDA, Bruna et al. Oxidative stress and inflammation in mucopolysaccharidosis type IVA patients treated with enzyme replacement therapy. BiochimicaetBiophysicaActa (BBA)-Molecular Basis of Disease, v. 1852, n. 5, p. 1012 1019, 2015.

GIESELMANN, Volkmar. Lysosomal storage diseases. BiochimicaetBiophysicaActa (BBA)-Molecular Basis of Disease, v. 1270, n. 2-3, p. 103-136, 1995.

HALLIWELL, B.; GUTTERIDGE, J. M. C. Cellular responses to oxidative stress: adaptation, damage, repair, senescence and death. Free radicals in biology and medicine, v. 4, p. 187 267, 2007.

HALLIWELL, Barry. Antioxidant defence mechanisms: from the beginning to the end (of the beginning). Free radical research, v. 31, n. 4, p. 261-272, 1999.

HALLIWELL, Barry. Oxidative stress and neurodegeneration: where are we now? Journalofneurochemistry, v. 97, n. 6, p. 1634-1658, 2006.

INTERFARMA, 2018. Doenças Raras: A urgência do acesso à saúde. Disponível em: https://www.interfarma.org.br/public/ files/biblioteca/doencas-raras--a-urgencia-do-acesso-a-saude-interfarma.pdf. Acessodia 05/11/2020.

LAMPE, Christina et al. Mucopolysaccharidoses and other lysosomal storage diseases. Rheumatic Disease Clinics, v. 39, n. 2, p. 431-455, 2013.

LEVINE, Rodney L. et al. [49] Determination of carbonyl content in oxidatively modified proteins. In: Methods in enzymology. Academic Press, 1990. p. 464-478. 
LOWRY, Oliver H. et al. Protein measurement with the Folin phenol reagent. J. biol. Chem, v. 193, n. 1, p. 265-275, 1951.

MELLO, Alexandre Silva et al. Oxidative stress parameters of Gaucher disease type I patients. Molecular geneticsandmetabolismreports, v. 4, p. 1-5, 2015.

OLIVEIRA, Monique Cristine de; SCHOFFEN, João Paulo Ferreira. Oxidative stress action in cellular aging. Brazilian Archives of Biology and Technology, v. 53, n. 6, p. 1333-1342, 2010.

PEREIRA, Catia S. et al. Invariant natural killer T cells are phenotypically and functionally altered in Fabry disease. Molecular genetics and metabolism, v. 108, n. 4, p. 241-248, 2013.

PLATT, Frances M. Sphingolipidlysosomal storage disorders. Nature, v. 510, n. 7503, p. 68, 2014.

ROZENFELD, Paula; FERIOZZI, Sandro. Contribuição das vias inflamatórias para a patogênese da doença de Fabry. Mol GenetMetab. Novembro de 2017; 122 (3): 19-27. doi: 10.1016 / j.ymgme.2017.09.004. Epub 13 de setembro de 2017. PMID: 28947349.

SALVADOR, MIRIAM; HENRIQUES, JOAO ANTONIO PEGAS. Radicais livres e a resposta celular ao estresse oxidativo. Editora da ULBRA, 2004.pp 35-36
SHEN, Jin-Song et al. Globotriaosylceramide induces oxidative stress and up-regulates cell adhesion molecule expression in Fabry disease endothelial cells. Molecular genetics and metabolism, v. 95, n. 3, p. 163-168, 2008.

SKOOG, WILLIAM A.; BECK, WILLIAM S. Studies on the fibrinogen, dextran and phytohemagglutinin methods of isolating leukocytes. Blood, v. 11, n. 5, p. 436-454, 1956.

TERMAN, Alexei et al. Lysosomallabilization. IUBMB life, v. 58, n. 9 , p. $531-539,2006$

VALKO, Marian et al. Free radicals and antioxidants in normal physiological functions and human disease. The international journal of biochemistry \& cell biology, v. 39, n. 1, p. 44-84, 2007.

VASCONCELOS, S.M.L, et al. Espécies reativas de oxigênio e de nitrogênio, antioxidantes e marcadores de dano oxidativo em sangue humano: principais métodos analíticos para sua determinação. Química Nova, v.30, n.5, p.1323-1338, 2007.

ZUGNO, Alexandra I. et al. Guanidinoacetate decreases antioxidant defenses and total protein sulfhydryl content in striatum of rats. Neurochemical research, v. 33, n. 9, p. 1804-1810, 2008.

WILLS E.D., Mechanism of lipid peroxide formation in animal tissues, Biochem. J. 99 (1996) 667-676. 